\title{
Proteinase-Activated Receptors in The Nervous System: Physiological and Pathological Aspects
}

\author{
Atefeh Aminian ${ }^{1}$, Farshid Noorbakhsh ${ }^{2 *}$ \\ ${ }^{1}$ Department of Pharmacology, Arak University of Medical Sciences, Arak, Iran \\ ${ }^{2}$ Department of Immunology, Tehran University of Medical Sciences, Tehran, Iran
}

\section{A BSTRACT}

Introduction: Proteinase-activated receptors (PARs), a family of four G protein-coupled receptors, are characterized by their unique activation mechanism which involves the proteolytic unmasking of a tethered ligand. To date, four PARs receptors have been discovered in human and mammals. All four members of the PARs family are expressed in the nervous system, where they have been shown to affect neural cell morphology, proliferation, and function. Furthermore, PARs play significant roles in degenerative and neuroinflammatory diseases, including Alzheimer's disease, multiple sclerosis, HIV-associated dementia, and s troke. The widespread distribution of PARs in the nervous system and their potential roles in different disorders make them attractive therapeutic targets for neurological diseases. Conclusion: In this review we summarize the roles of PARs in the central and peripheral nervous systems in the physiological setting as well as in pathological conditions.

*Corresponding Author: Farshid Noorbakhsh

E-mail:f-noorbakhsh@sina.tums.ac.ir 


\title{
كيرندهاى فعال شونده با يروتئيناز در سيستم عصبى، جنبههاى فيزيولوزى و پاتولوزى
}

\author{
عاطفه امينيان'، فرشيد نوربخش " \\ اكروه فارماكولوزى، دانشكاه علوم يزشكى اراك، اراك، ايران

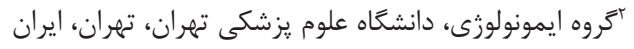

( اطلاعات مقاله:

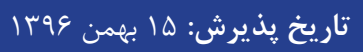

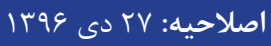

تاريخ دريافت: 9 آذر عهسا

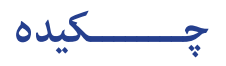

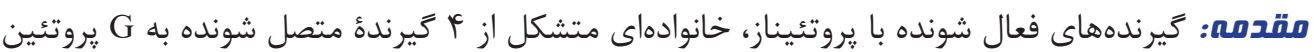

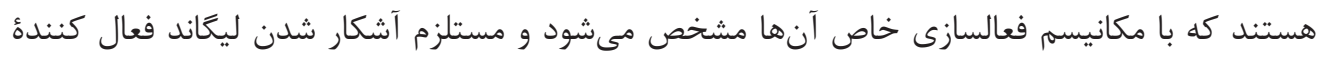

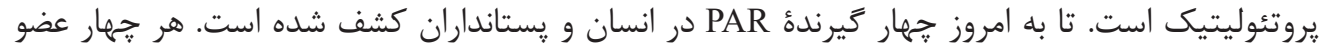

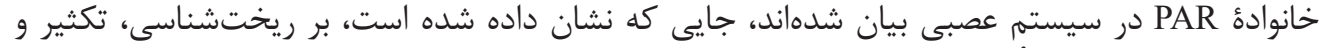

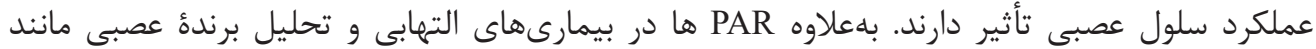

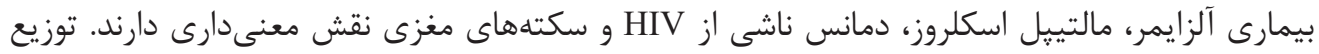

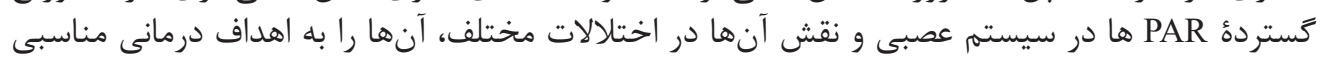

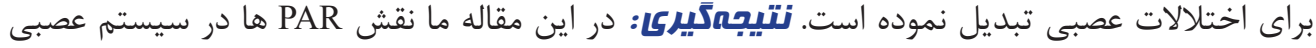

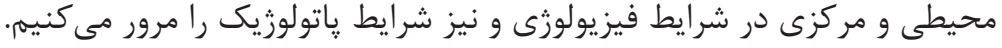

ا

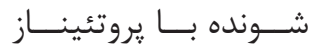

r.

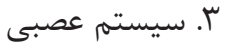

مركزى

" نويسنده مسئول: فرشيد نوربخش

آدرس الكترونيكى: f-noorbakhsh@sina.tums.ac.ir 


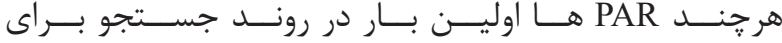

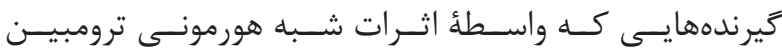

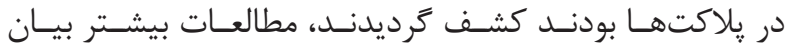

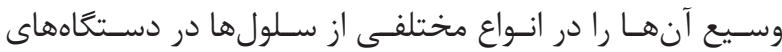

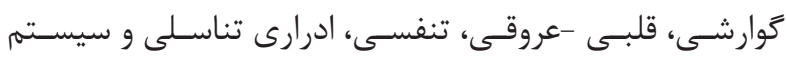

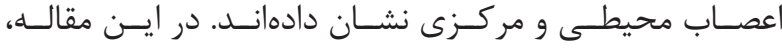

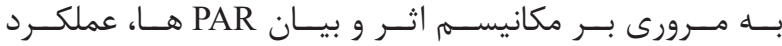

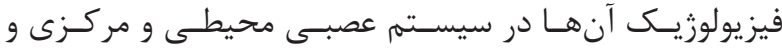

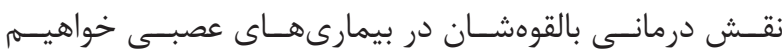

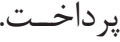

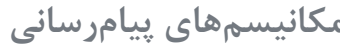

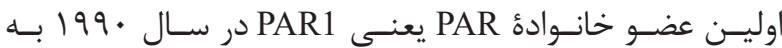

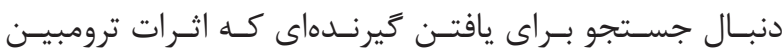

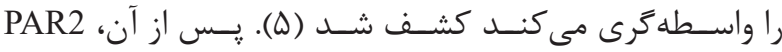

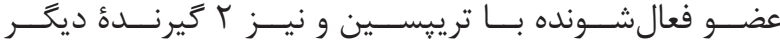

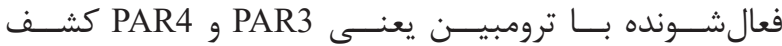

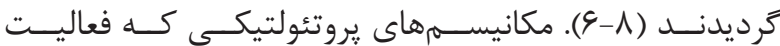

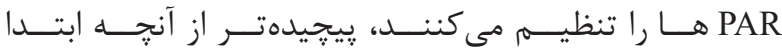

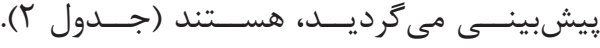

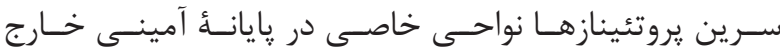

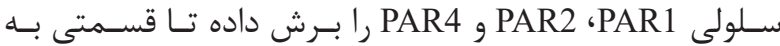

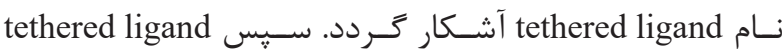

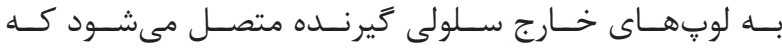

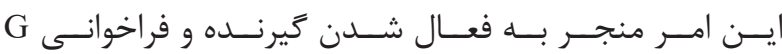

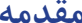

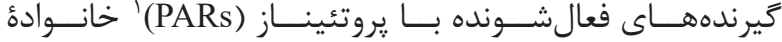

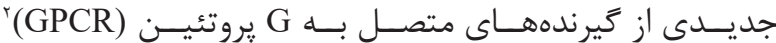

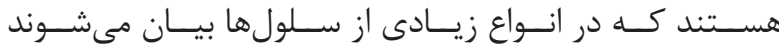

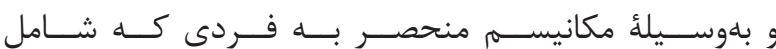

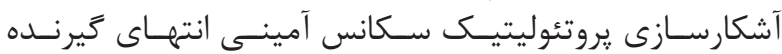

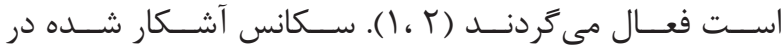

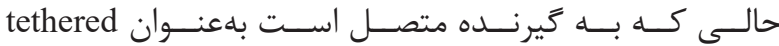
ligand

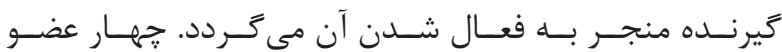

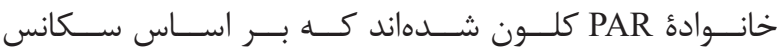
tethered ligand

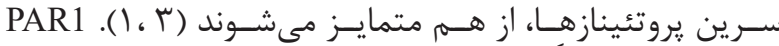

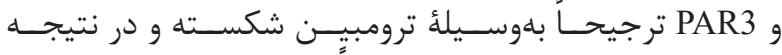

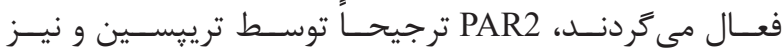

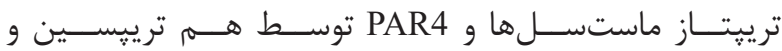

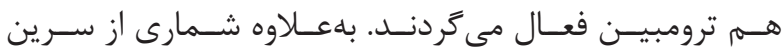

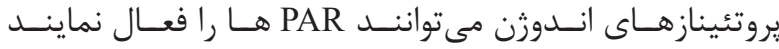

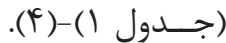

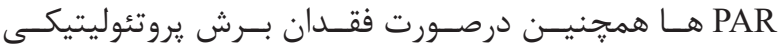

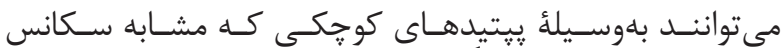

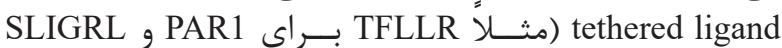

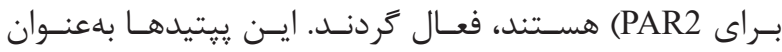

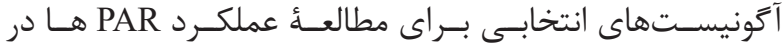

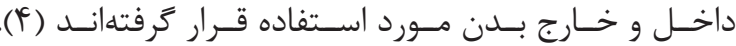

\begin{tabular}{|c|c|c|c|c|}
\hline PAR4 & PAR3 & PAR2 & PARI & \\
\hline 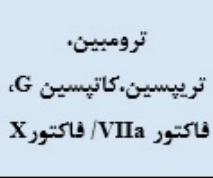 & تروعبين. ترييسين & 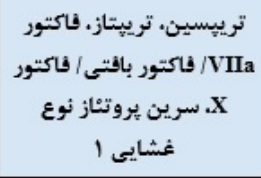 & 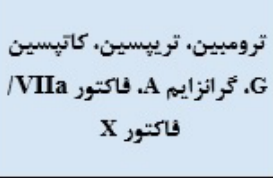 & كبنئدئن هاى فعال \\
\hline GYPGQV & TFRGAP & SLIGKV & SFLLR & $\begin{array}{c}\text { سكانس lethered } \\
\text { ligand }\end{array}$ \\
\hline $\begin{array}{l}\text { GYPGQV-NH2, } \\
\text { GYPGKF-NH2, } \\
\text { AYPGKF-NH2 }\end{array}$ & & $\begin{array}{c}\text { SLIGKV-NH2, } \\
\text { SLIGRL-NH2, trans- } \\
\text { cinnamoyl-LIGRLO- } \\
\text { NH2 } \\
\end{array}$ & TFRIFD, TFLLR-NH2 & يبتيد فعال كنتده \\
\hline $\mathrm{Ca}^{2+}$ & ERK1/2 & $\begin{array}{c}\mathrm{Gaq} / \mathrm{Ca}^{2+}, \\
\text { Gal2/13-Rho, MAPK, } \\
\text { ERK1/2, } \beta \text {-arrestin, } \\
\text { Akt, Gai and } \\
\text { Gas/cAMP } \\
\end{array}$ & 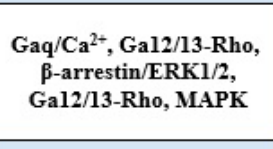 & مسيرهاى ييامرسانى \\
\hline آميخدال. تالاعوس و هيبو. & 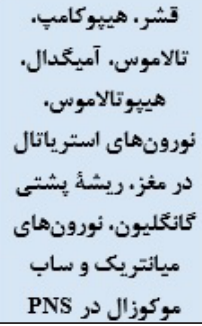 & 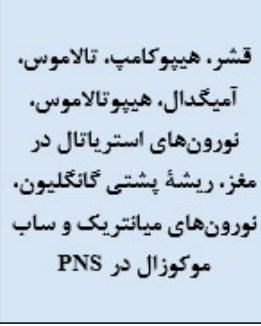 & 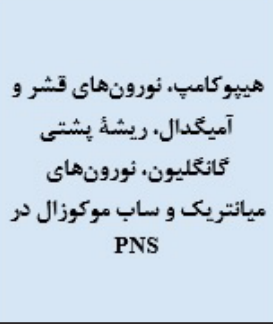 & سواحى موجود PARs در \\
\hline
\end{tabular}




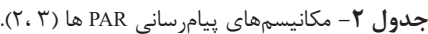

\begin{tabular}{|c|c|c|}
\hline توضيحات & مثال & $\begin{array}{l}\text { مكانيسمهاى ٪يامرسانى } \\
\text { PARs }\end{array}$ \\
\hline 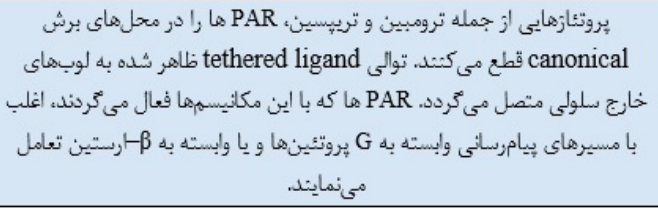 & فعال شدن PAR3 توسط ترومبين & $\begin{array}{l}\text { فعال شدن استاندارد } \\
\text { (canonical) }\end{array}$ \\
\hline 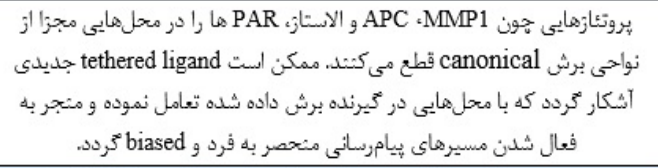 & فعال شدن PAR1 توسط الاستار & biased agonism \\
\hline 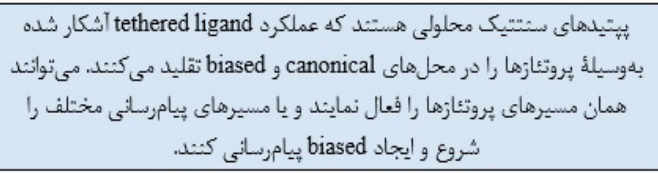 & $\begin{array}{l}\text { فعال شدن PAR2 توسط } \\
\text { SLIGKV-NH2 }\end{array}$ & فعال شدن توسط يتيدهاى فعال كنبنه \\
\hline 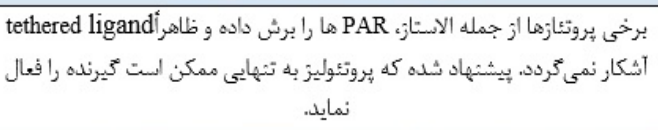 & فعال شدن PAR2 توسط الاستاز & 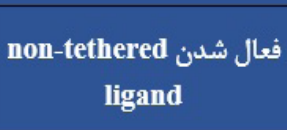 \\
\hline 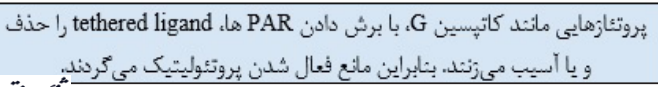 & $\begin{array}{c}\text { غير فعال نمونن PAR2 توسط } \\
\text { كاتيسين }\end{array}$ & Proteolytic disarming \\
\hline
\end{tabular}

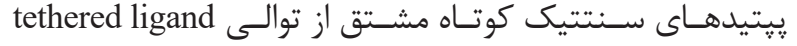

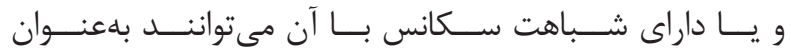

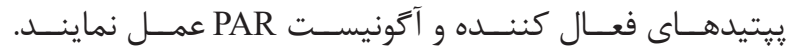

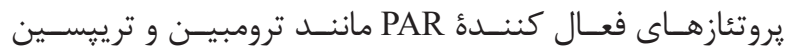

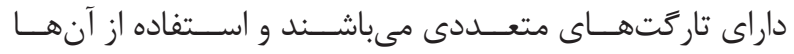

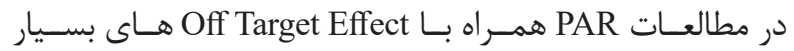

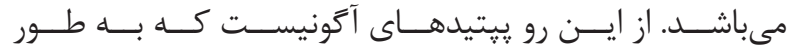

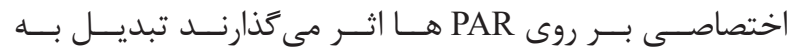

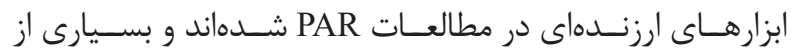

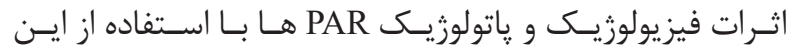

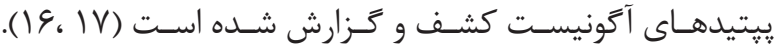

بيان PAR ها در سيستم عصبى مركزى و محيطى هـر جهـار PAR بــه طـور وسـيعى در نواحسى مختلف سيسـتم

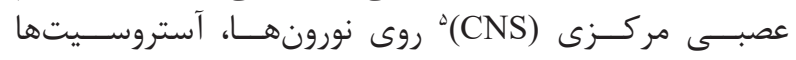

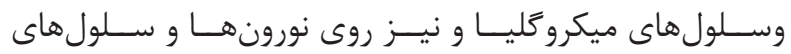

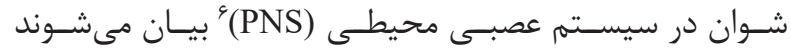

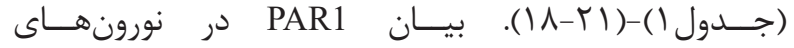

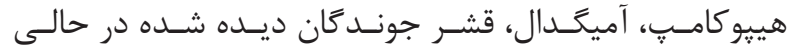

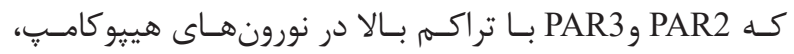

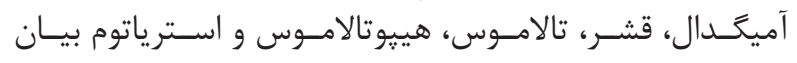

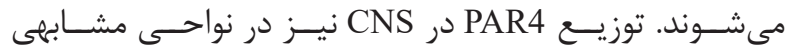

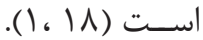

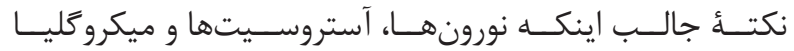

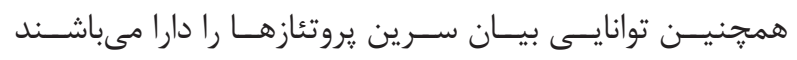

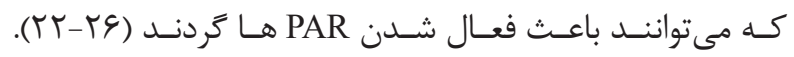

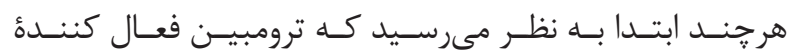

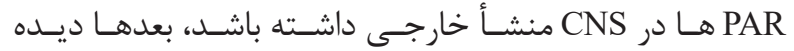

${ }^{3}$ Signaling

${ }^{4}$ Mitogen-activated protein kinase

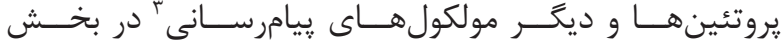

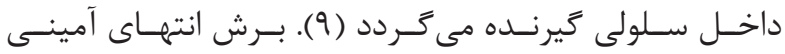

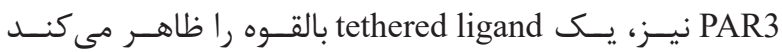

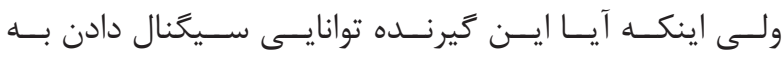

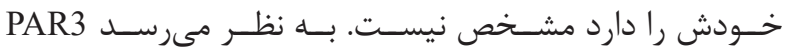

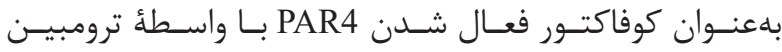

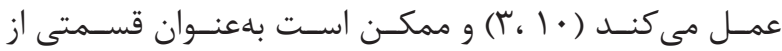

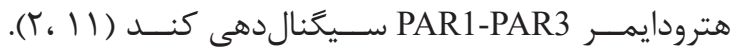

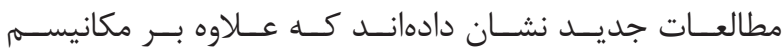

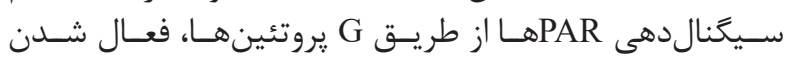

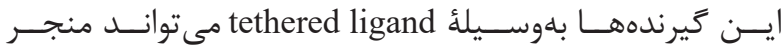

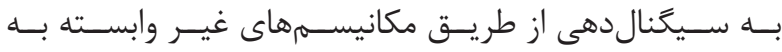

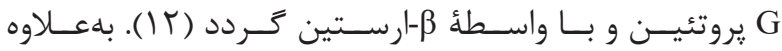

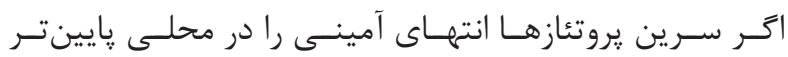

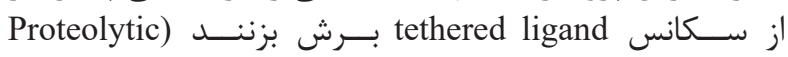

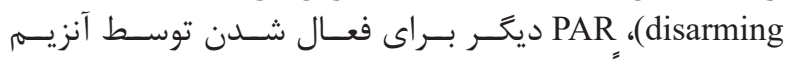

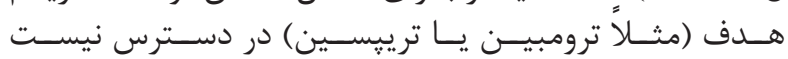

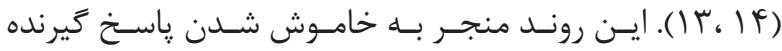

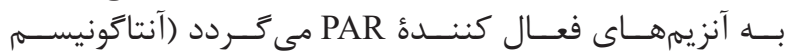

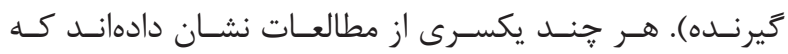

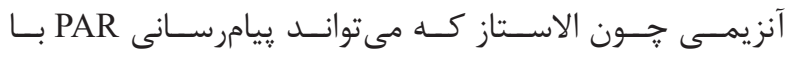

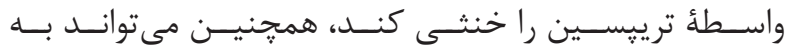

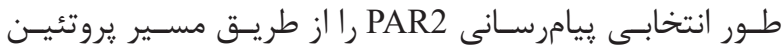

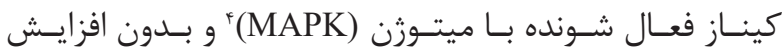

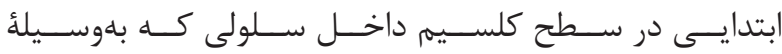
tethered ligand

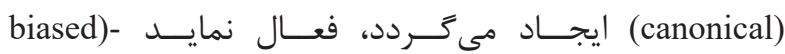
.(Y. IQ)-(agonism

${ }^{5}$ Central nervous system

${ }^{6}$ Peripheral nervous system 


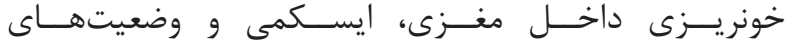

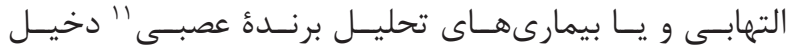

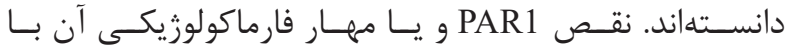

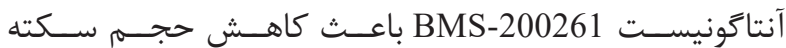

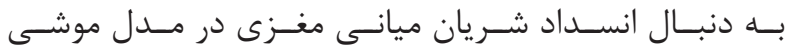

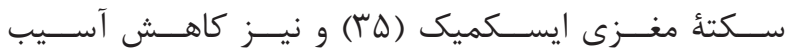

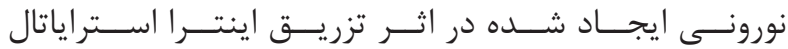

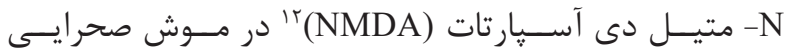

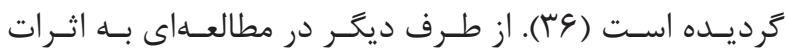

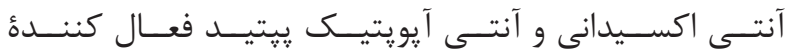
PAR1 (SFLLRN-NH2)

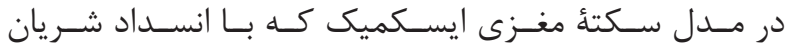

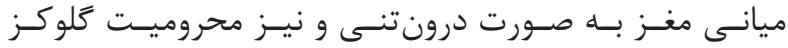

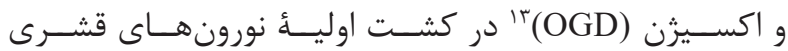

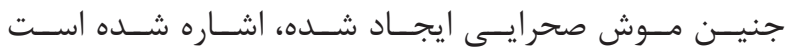

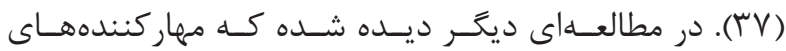

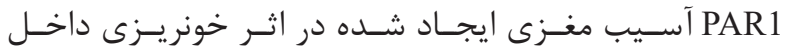

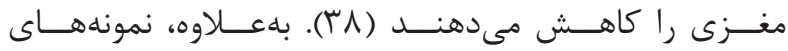

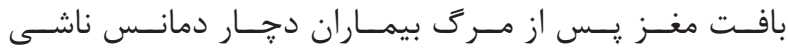

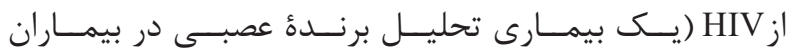

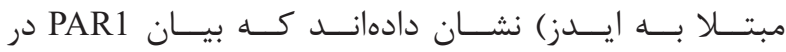

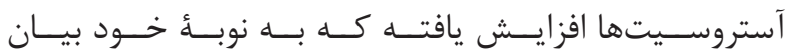

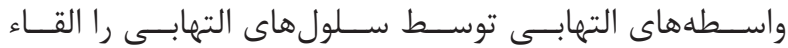

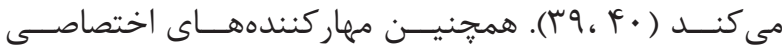

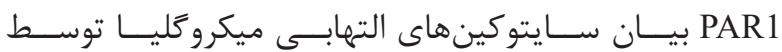
d-synuclein

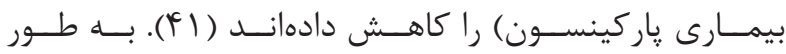

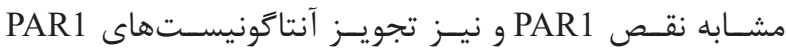

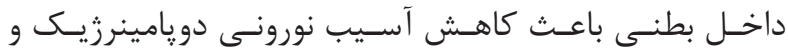

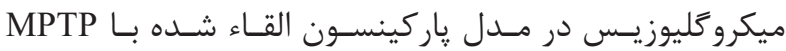

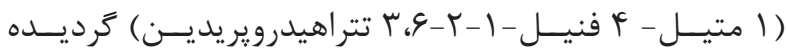

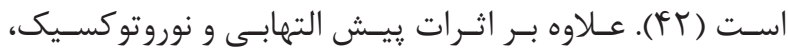

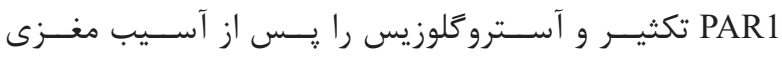

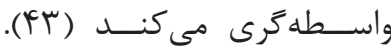

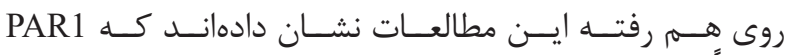

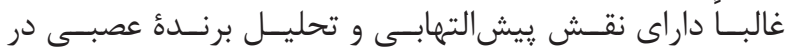

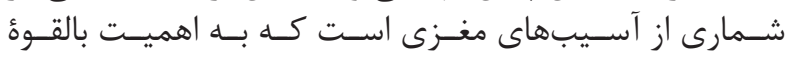

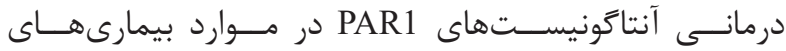

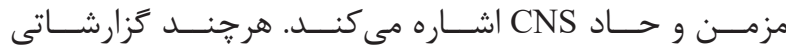

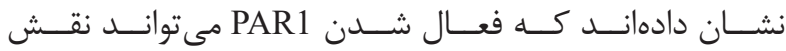

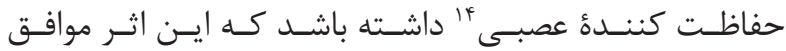

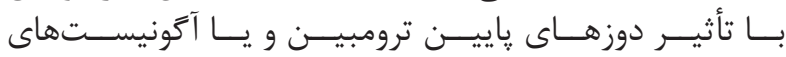

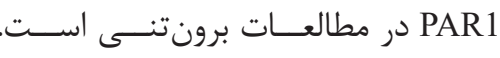

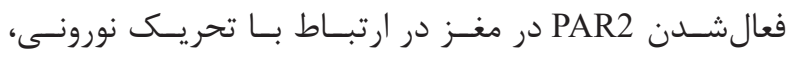

${ }^{7}$ Protease nexin-1

${ }^{8}$ Antithrombin 3

${ }^{9}$ Blood brain barrier

${ }^{10}$ Morphology

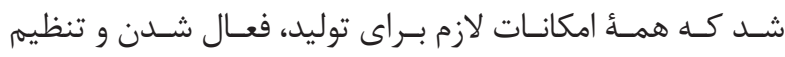

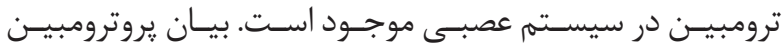

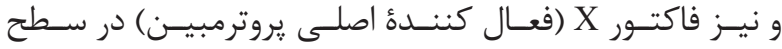

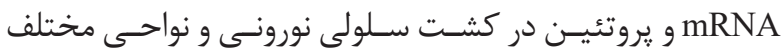

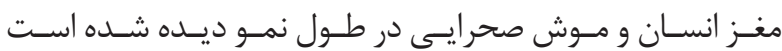

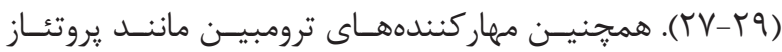

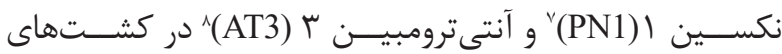

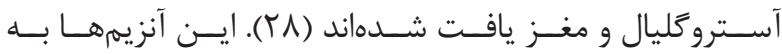

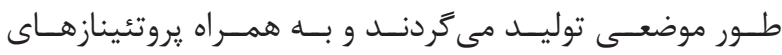

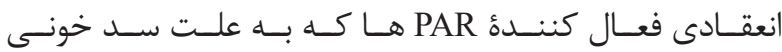

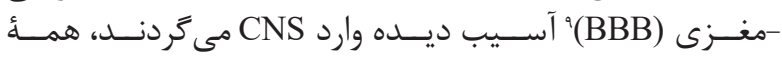

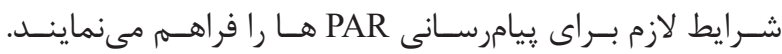

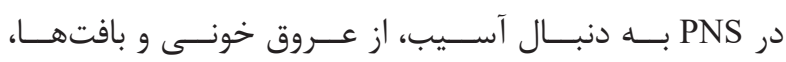

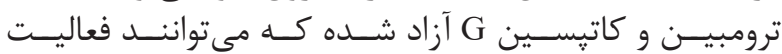

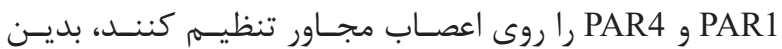

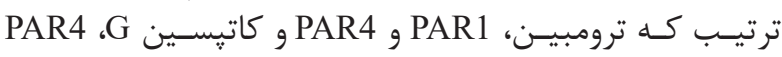

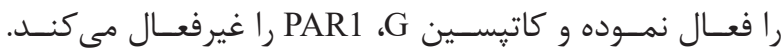

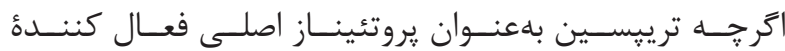

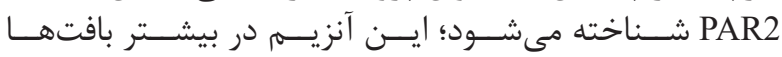

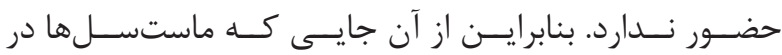

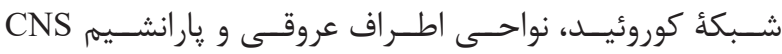

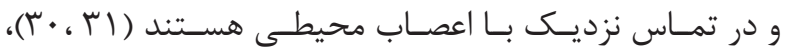

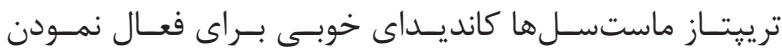
PAR2

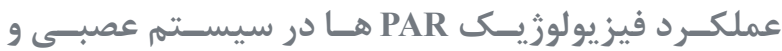

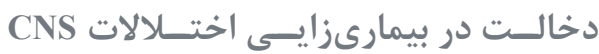

اثـر PAR هـا بــر روى نمــو، تكثيــر و زنـده مانـدن سـلول هاى

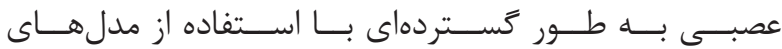

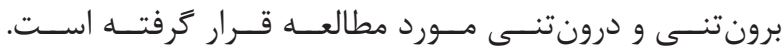

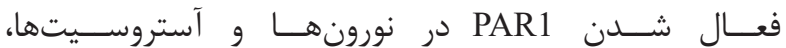

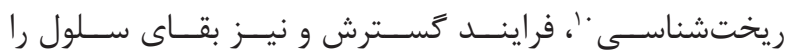

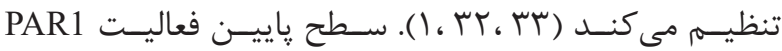

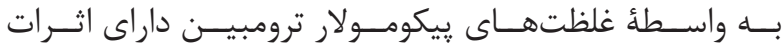

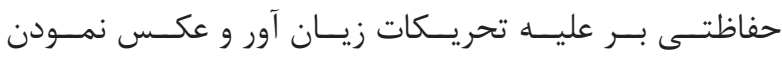

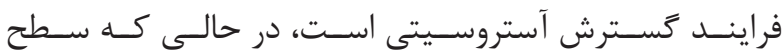

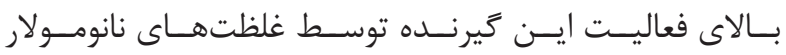

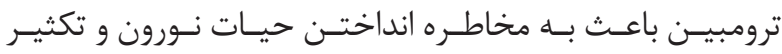

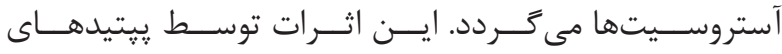

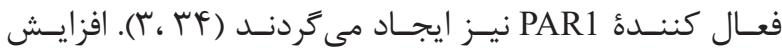

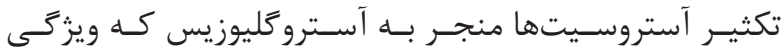

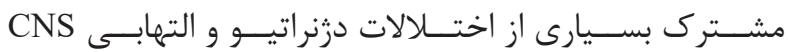

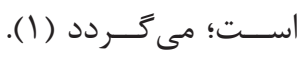

مطالعــات بســيارى بيامرســانى PAR1 را در بيمارىزايسىى
${ }^{11}$ Neurodegenerative
${ }^{12} \mathrm{~N}$-Methyl-D-aspartate
${ }^{13}$ Oxygen-glucose deprivation
${ }^{14}$ Neuroprotective 


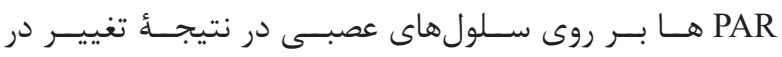

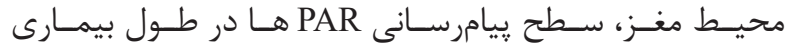

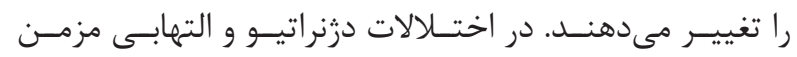

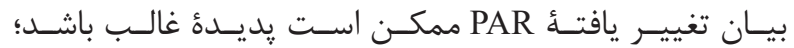

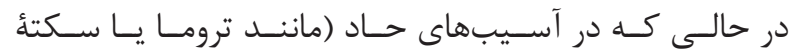

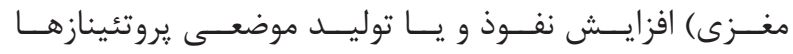

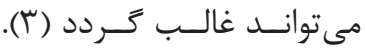

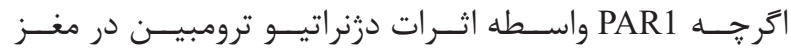

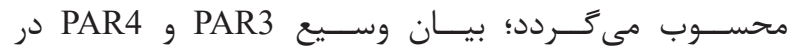

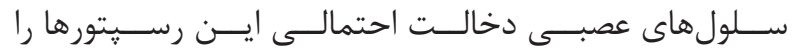

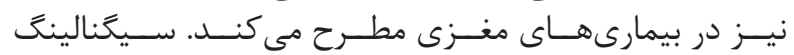

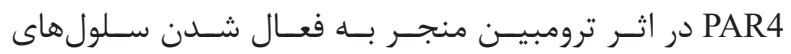

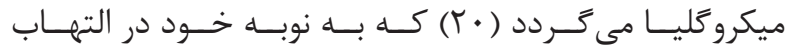

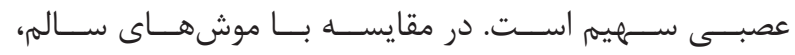

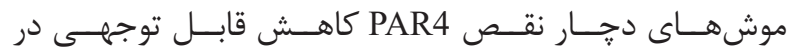

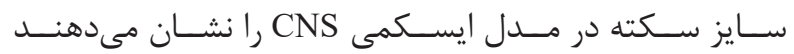

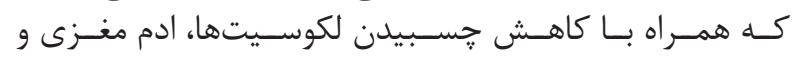

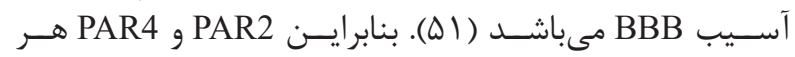

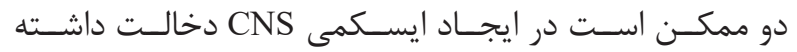

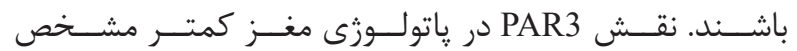

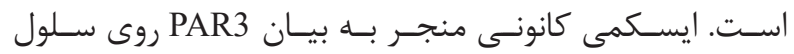

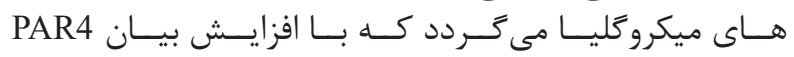

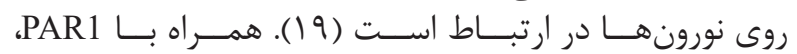

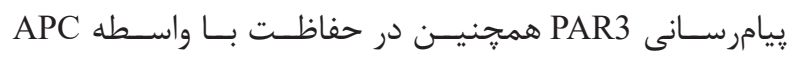

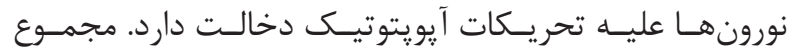

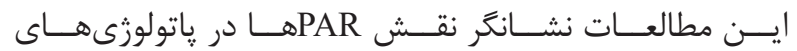

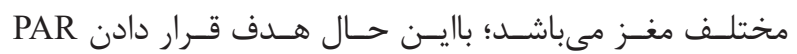

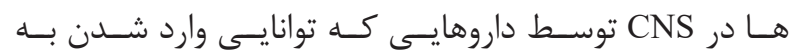

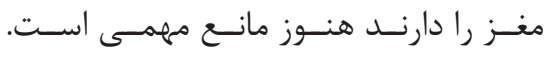

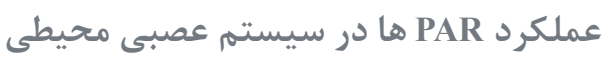

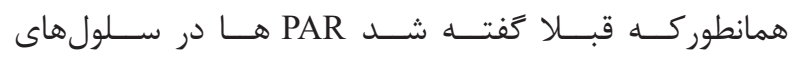

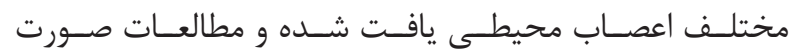

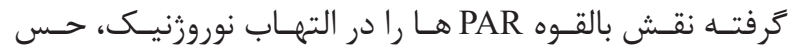

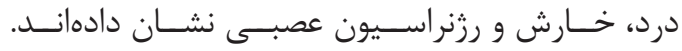

PAR1 و PAR2 در نورونهــاى اوليسـه آوران نخـــاع بيـان

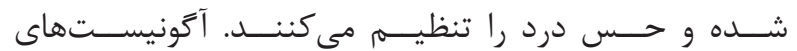

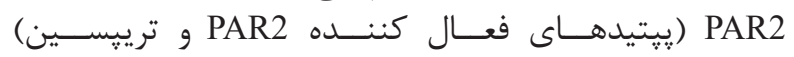

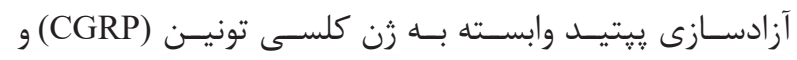

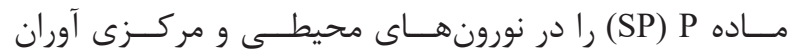

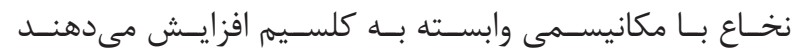

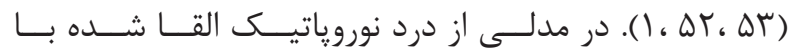

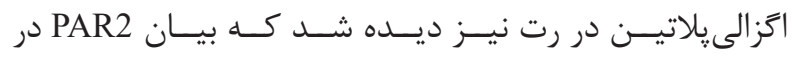

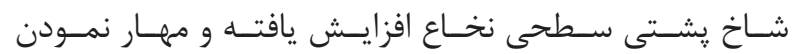
transient receptor potential vanilloid 1(TRPV1) و

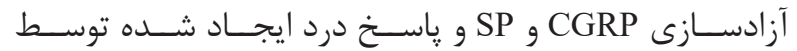

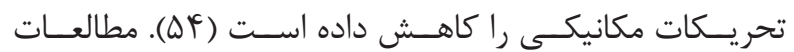

${ }^{15}$ Monocytoid

${ }^{16}$ Experimental autoimmune encephalomyelitis

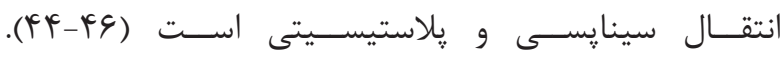

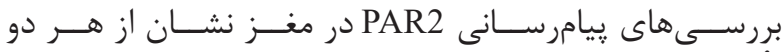

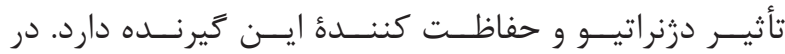

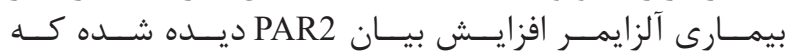

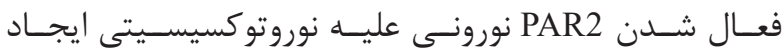

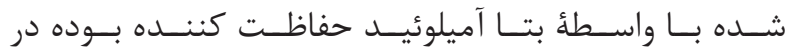

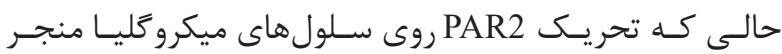

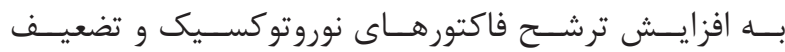

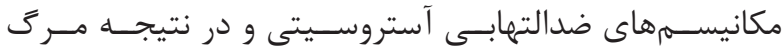

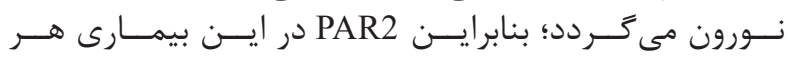

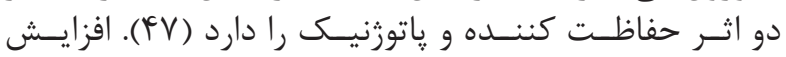

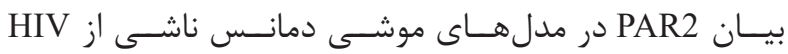

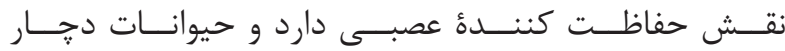

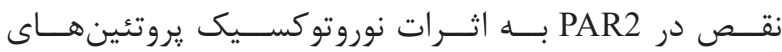

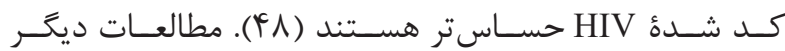

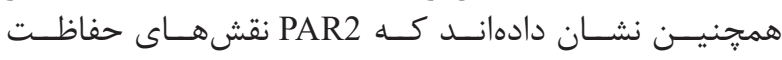

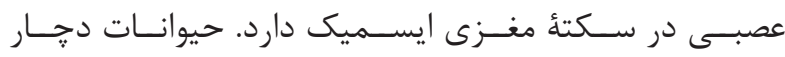

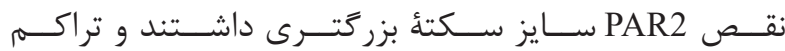

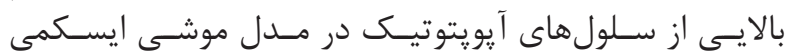

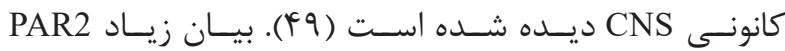

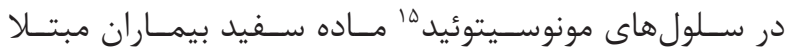

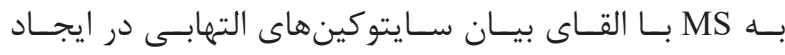

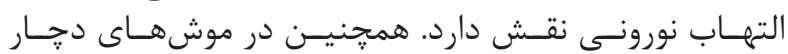

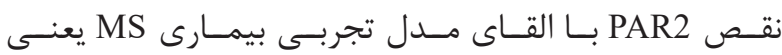

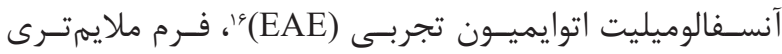

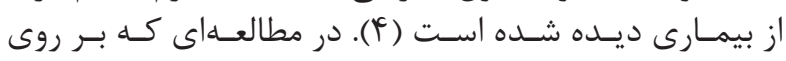

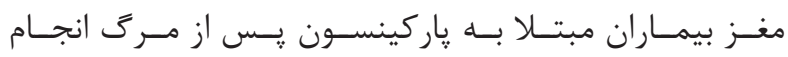

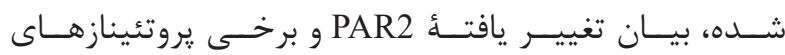

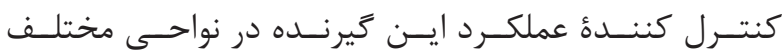

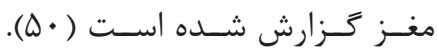

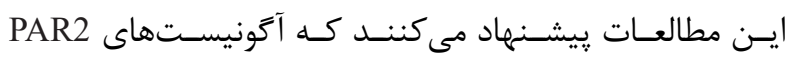

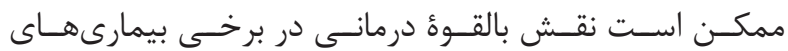

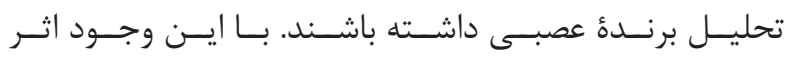

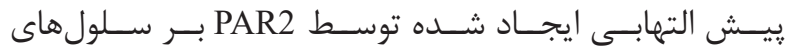

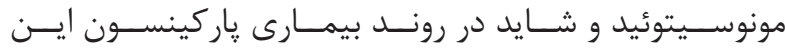

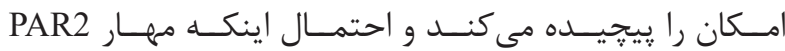

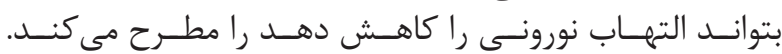

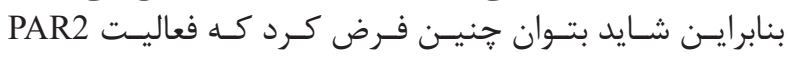

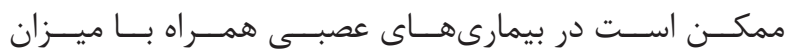

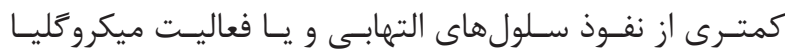

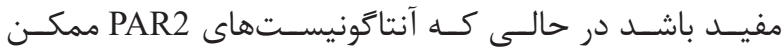

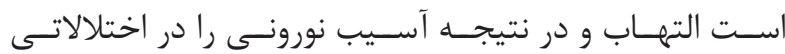

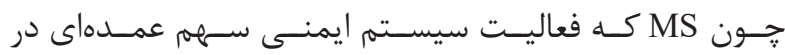

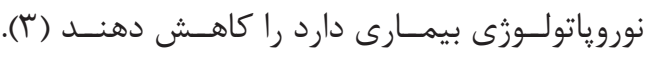

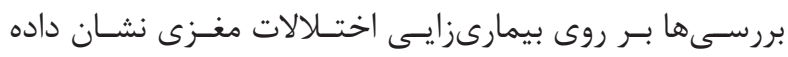

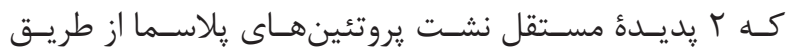

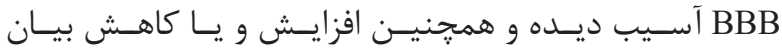




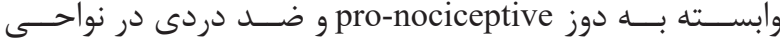
احشـايى و سـوماتيك ايجــاد كنــــد (هو). نتيجه گيرى

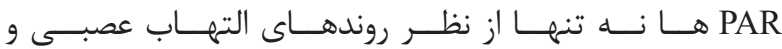

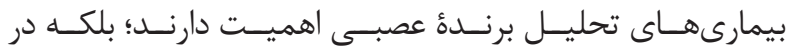

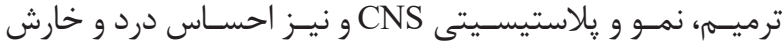

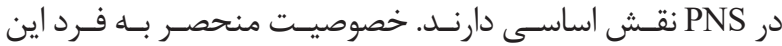

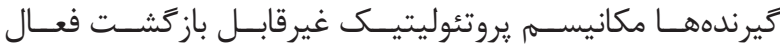

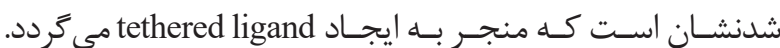

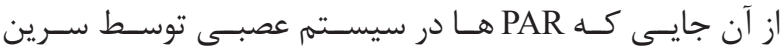

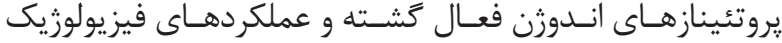

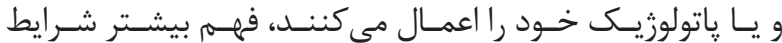

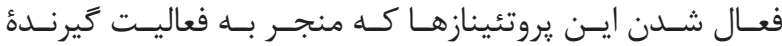

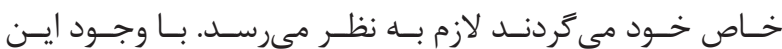

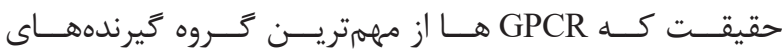

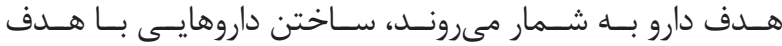

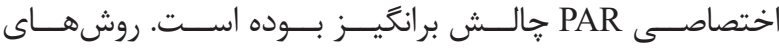

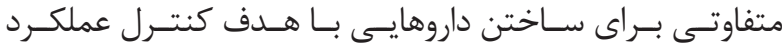

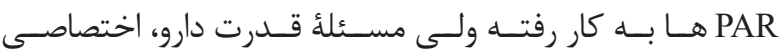

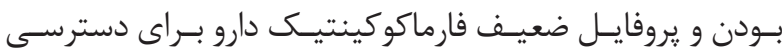

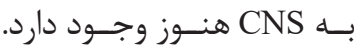

1. Noorbakhsh F, Vergnolle N, Hollenberg MD, Power C. Proteinase-activated receptors in the nervous system. Nat Rev Neurosci. 2003;4(12): 981-90.

2. Zhao P, Metcalf M, Bunnett NW. Biased signaling of protease-activatedreceptors. Front Endocrinol (Lausanne). 2014; 5: 67. doi: 10.3389/fendo.2014.00067.

3. Ramachandran R, Noorbakhsh F, Defea K, Hollenberg MD. Targeting proteinase-activated receptors: therapeutic potential and challenges. Nat Rev Drug Discov. 2012;11(1): 69-86.

4. Noorbakhsh F, Tsutsui S, Vergnolle N, Boven LA, Shariat N, Vodjgani M, et al. Proteinase-activated receptor 2 modulates neuroinflammation in experimental autoimmune encephalomyelitis and multiple sclerosis. J Exp Med. 2006; 203(2): 425-35.

5. Vu TK, Hung DT, Wheaton VI, Coughlin SR. Molecular cloning of a functional thrombin receptor reveals a novel proteolytic mechanism of receptor activation. Cell. 1991; 64(6): 1057-68.

6. Rasmussen UB, Vouret-Craviari V, Jallat S, Schlesinger Y, Pages G, Pavirani A, et al. cDNA cloning and expression of a hamster alpha-thrombin receptor

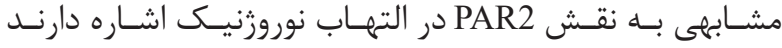

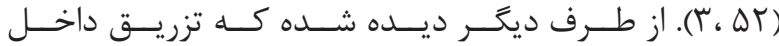

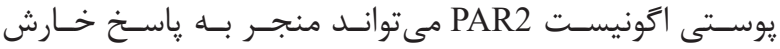

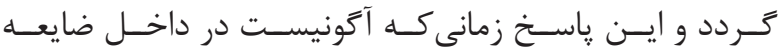

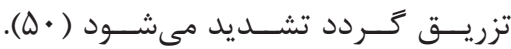

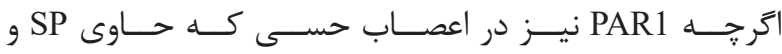

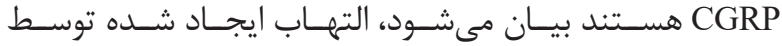

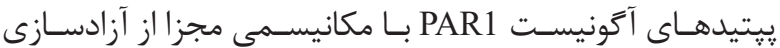

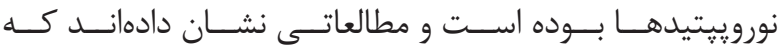

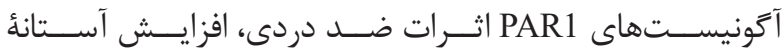

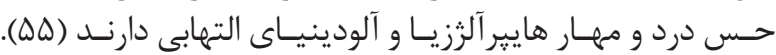

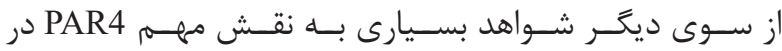

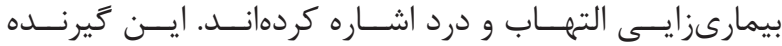

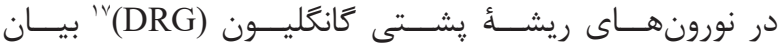

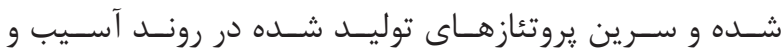

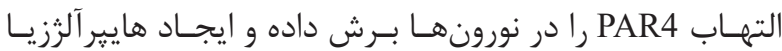

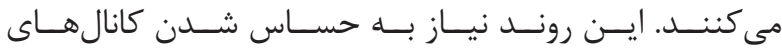

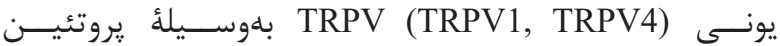
كينــاز C و مسـير زيامرسـانى MAPK/ERK و نيـز افزايسـش

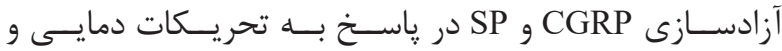

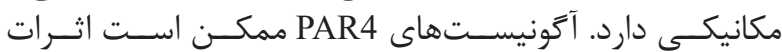

منابع

coupled to Ca2+ mobilization. FEBS Lett. 1991; 288(12): $123-8$.

7. Nystedt S, Emilsson K, Wahlestedt C, Sundelin J. Molecular cloning of a potential proteinase activated receptor. Proc Natl Acad Sci U S A. 1994; 91(20): 9208-12.

8. Bohm SK, Kong W, Bromme D, Smeekens SP, Anderson DC, Connolly A, et al. Molecular cloning, expression and potential functions of the human proteinase-activated receptor-2. Biochem J. 1996; 314(3): 1009-16.

9. Adams MN, Ramachandran R, Yau MK, Suen JY, Fairlie DP, Hollenberg MD, et al. Structure, function and pathophysiology of protease activated receptors. Pharmacol Ther. 2011; 130(3): 248-82.

10. Nakanishi-Matsui M, Zheng YW, Sulciner DJ, Weiss EJ, Ludeman MJ, Coughlin SR. PAR3 is a cofactor for PAR4 activation by thrombin. Nature.2000; 404(6778): 609-13.

11. McLaughlin JN, Patterson MM, Malik AB. Proteaseactivated receptor-3 (PAR3) regulates PAR1 signaling by receptor dimerization. Proc Natl Acad Sci U S A. 2007; 


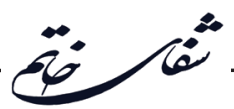

104(13): 5662-7.

12. Defea K. Beta-arrestins and heterotrimeric G-proteins: collaborators and competitors in signal transduction. Br J Pharmacol. 2008; 153(1): S298-309.

13. Dulon S, Cande C, Bunnett NW, Hollenberg MD, Chignard M, Pidard D. Proteinase-activated receptor-2 and human lung epithelial cells: disarming by neutrophil serine proteinases. Am J Respir Cell Mol Biol. 2003; 28(3): 339-46.

14. Renesto P, Si-Tahar M, Moniatte M, Balloy V, Van Dorsselaer A, Pidard D, et al. Specific inhibition of thrombin-induced cell activation by the neutrophil proteinases elastase, cathepsin G, and proteinase 3: evidence for distinct cleavage sites within the amino terminal domain of the thrombin receptor. Blood. 1997; 89(6): 1944-53.

15. Ramachandran R, Mihara K, Chung H, Renaux B, Lau CS, Muruve DA, et al. Neutrophil elastase acts as a biased agonist for proteinase-activated receptor-2 (PAR2). J Biol Chem. 2011; 286(28): 24638-48.

16. Scarborough RM, Naughton MA, Teng W, Hung DT, Rose J, Vu TK, et al. Tethered ligand agonist peptides. Structural requirements for thrombin receptor activation reveal mechanism of proteolytic unmasking of agonist function. J Biol Chem. 1992 ;267(19): 13146-9.

17. Hollenberg MD, Saifeddine M, al-Ani B, Kawabata A. Proteinase-activated receptors: structural requirements for activity, receptor cross-reactivity, and receptor selectivity of receptor-activating peptides. Can J Physiol Pharmacol. 1997; 75(7): 832-41.

18. Striggow F, Riek-Burchardt M, Kiesel A, Schmidt W, Henrich-Noack P, Breder J, et al. Four different types of protease-activated receptors are widely expressed in the brain and are up-regulated in hippocampus by severe ischemia. Eur J Neurosci. 2001; 14(4): 595-608.

19. Henrich-Noack P, Riek-Burchardt M, Baldauf $\mathrm{K}$, Reiser G, Reymann KG. Focal ischemia induces expressionof protease-activated receptor1 (PAR1) and PAR3 on microglia and enhances PAR4 labeling in the penumbra. Brain Res. 2006; 1070(1): 232-41.

20. Suo Z, Wu M, Citron BA, Gao C, Festoff BW. Persis tent protease-activated receptor 4 signaling mediates thrombin-induced microglial activation. J Biol Chem. 2003; 278(33): 31177-83.

21. Wang H, Ubl JJ, Reiser G. Four subtypes of proteaseactivated receptors, co-expressed in rat astrocytes, evoke different physiological signaling. Glia. 2002; 37(1):
53-63.

22. Scarisbrick IA, Isackson PJ, Ciric B, Windebank AJ, Rodriguez M. MSP, a trypsin-like serine protease, is abundantly expressed in the human nervous system. $\mathrm{J}$ Comp Neurol. 2001; 431(3): 347-61.

23. Bernett MJ, Blaber SI, Scarisbrick IA, Dhanarajan P, Thompson SM, Blaber M. Crystal structure and biochemical characterization of human kallikrein 6 reveals that a trypsin-like kallikrein is expressed in the central nervous system. J Biol Chem. 2002; 277(27): 24562-70.

24. Sokolova E, Reiser G. Prothrombin/thrombin and the thrombin receptors PAR-1 and PAR-4 inthe brain: localization, expression and participation in neurodegenerative diseases. Thromb Haemost. 2008; 100(4): 576-81.

25. Yoshida S, Shiosaka S. Plasticity-related serine proteases in the brain (review). Int J Mol Med. 1999; 3(4): 405-9.

26. Turgeon VL, Houenou LJ. The role of thrombin-like (serine) proteases in the development, plasticity and pathology of the nervous system. Brain Res Rev. 1997; 25(1): 85-95.

27. Deschepper CF, Bigornia V, Berens ME, Lapointe MC. Production of thrombin and antithrombin III by brain and astroglial cell cultures. Molecular Brain Research. 1991; 11(3-4): 355-8

28. Dihanich M, Kaser M, Reinhard E, Cunningham D, Monard D. Prothrombin mRNA is expressed by cells of the nervous system. Neuron. 1991; 6(4): 575-81.

29. Shikamoto Y, Morita T. Expression of factor $X$ in boththe rat brain and cells of the central nervous system. FEBS Lett. 1999; 463(3): 387-9.

30. Theoharides TC. Mast cells: the immune gate to the brain. Life Sci. 1990; 46(9): 607-17.

31. Stead RH, Tomioka M, Quinonez G, Simon GT, Felten SY, Bienenstock J. Intestinal mucosal mast cells in normal and nematode-infected rat intestines are in intimate contact with peptidergic nerves. Proc Natl Acad Sci U S A. 1987; 84(9): 2975-9.

32. Luo W, Wang Y, Reiser G. Protease-activated receptors in the brain: receptor expression, activation, and functions in neurodegeneration and neuroprotection. Brain Res Rev. 2007; 56(2): 331-45.

33. Tanaka M, Yoneyama M, Shiba T, Yamaguchi 
T, Ogita K. Protease-activated receptor-1 negatively regulates proliferation of neural stem/progenitor cells derived from the hippocampal dentate gyrus of the adult mouse. J Pharmacol Sci. 2016; 131(3): 162-71.

34. Beecher KL, Andersen TT, Fenton JW, Festoff BW. Thrombin receptor peptides induce shape change in neonatal murine astrocytes in culture. J Neurosci Res. 1994; 37(1)108-15

35. Junge CE, Sugawara T, Mannaioni G, Alagarsamy $\mathrm{S}$, Conn PJ, Brat DJ, et al. The contribution of proteaseactivated receptor 1 to neuronal damage caused by transient focal cerebralischemia. Proc Natl Acad Sci U S A. 2003; 100(22): 13019-24.

36. Hamill CE, Mannaioni G, Lyuboslavsky P, Sastre AA, Traynelis SF. Protease-activated receptor 1-dependent neuronal damage involves NMDA receptor function. Exp Neurol. 2009; 217(1): 136-46.

37. Zhen X, Ng ES, Lam FF. Suppression of ischaemiainduced injuries in ratbrain by protease-activated receptor-1 (PAR-1) activating peptide. Eur J Pharmacol. 2016; 786: 36-46.

38. Xue M, Hollenberg MD, Demchuk A, Yong VW. Relative importance of proteinase-activated receptor-1 versusmatrixmetalloproteinases in intracerebral hemorrhage-mediated neurotoxicity in mice. Stroke. 2009; 40(6): 2199-204.

39. Acharjee S, Zhu Y, Maingat F, Pardo C, Ballanyi K, Hollenberg MD, et al. Proteinase-activated receptor-1 mediates dorsal root ganglion neuronal degeneration in HIV/AIDS. Brain. 2011; 134(11): 3209-21.

40. Boven LA, Vergnolle N, Henry SD, Silva C, Imai Y, Holden J, et al. Up-regulation of proteinaseactivated receptor 1 expression in astrocytes during HIV encephalitis. J Immunol. 2003; 170(5): 2638-46.

41. Lee EJ, Woo MS, Moon PG, Baek MC, Choi IY, Kim WK, et al. Alpha-synuclein activates microglia by inducing the expressions of matrix metalloproteinases and the subsequent activation of protease-activated receptor-1. J Immunol. 2010; 185(1): 615-23.

42. Hamill CE, Caudle WM, Richardson JR, Yuan $\mathrm{H}$, Pennell KD, Greene JG, et al. Exacerbation of dopaminergic terminal damage in a mouse model of Parkinson's disease by the G-protein-coupled receptor protease-activated receptor 1. Mol Pharmacol. 2007; 72(3): 653-64.

43. Nicole O, Goldshmidt A, Hamill CE, Sorensen SD,
Sastre A, Lyuboslavsky P, et al. Activation of proteaseactivated receptor-1 triggers astrogliosis after brain injury. J Neurosci. 2005; 25(17): 4319-29.

44. Gan J, Greenwood SM, Cobb SR, Bushell TJ. Indirect modulation of neuronal excitability and synaptic transmission in the hippocampus by activation of proteinase-activated receptor-2. BJP. 2011; 163(5): 984-94.

45. Lohman RJ, O’Brien TJ, Cocks TM. Proteaseactivated receptor- 2 regulates trypsin expression in the brain and protects against seizuresandepileptogenesis. Neurobiol Dis. 2008; 30(1): 84-93.

46. Lohman RJ, Jones NC, O’Brien TJ, Cocks TM. A regulatory role for protease-activated receptor- 2 in motivational learning in rats. Neurobiol Learn Mem. 2009; 92(3): 301-9.

47. Afkhami-Goli A, Noorbakhsh F, Keller AJ, Vergnolle $\mathrm{N}$, Westaway D, Jhamandas JH, et al. Proteinaseactivated receptor-2 exerts protective and pathogenic cell type-specific effects in Alzheimer's disease. J Immunol. 2007; 179(8): 5493-503.

48 Noorbakhsh F, Vergnolle N, McArthur JC, Silva C, Vodjgani M, Andrade-Gordon P, et al. Proteinaseactivated receptor-2 induction by neuroinflammation prevents neuronal deathduring HIV infection. J Immunol. 2005; 174(11): 7320-9.

49. Jin G, Hayashi T, Kawagoe J, Takizawa T, Nagata T, Nagano I, et al. Deficiency of PAR-2 gene increases acute focal ischemic brain injury. Journal of cerebral blood flow andmetabolism: J Cereb Blood Flow Metab. 2005; 25(3): 302-13.

50. Bar-Shavit R, Maoz M, Kancharla A, Jaber M, Agranovich D, Grisaru-Granovsky S, et al. Proteaseactivated receptors (PARs) in cancer: Novel biased signaling and targets for therapy. Methods Cell Biol. 2016; 132: 341-58.

51. Mao Y, Zhang M, Tuma RF, Kunapuli SP. Deficiency of PAR4 attenuates cerebral ischemia/reperfusion injury in mice. J Cereb Blood Flow Metab. 2010; 30(5): 1044-52.

52. Rothmeier AS, Ruf W. Protease-activated receptor 2 signaling in inflammation. Semin Immunopathol. 2012; 34(1): 133-49.

53. Steinhoff M, Vergnolle N, Young SH, Tognetto M, Amadesi S, Ennes HS, et al. Agonists of proteinaseactivated receptor 2 induce inflammation by a neurogenic mechanism. Nat Med. 2000; 6(2): 151-8. 


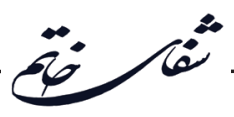

54. Chen K, Zhang ZF, Liao MF, Yao WL, Wang J, Wang XR. Blocking PAR2 attenuates oxaliplatin-induced neuropathic pain via TRPV1 and releases of substance $\mathrm{P}$ and CGRP in superficial dorsal horn of spinal cord. J Neurol Sci. 2015; 352(1-2): 62-7.

55. Asfaha S, Brussee V, Chapman K, Zochodne DW, Vergnolle N. Proteinase-activated receptor-1 agonis ts attenuate nociception in response to noxious stimuli. $\mathrm{Br}$ J Pharmacol. 2002; 135(5): 1101-6.

56. Bao Y, Gao Y, Yang L, Kong X, Zheng H, Hou W, et al. New insights into protease-activated receptor 4 signaling pathways in the pathogenesis of inflammation and neuropathic pain: a literature review. Channels. 2015; 9(1): 5-13. 